uptake pattern was significantly lower in HAVS than in primary RP.

Conclusions There were significant differences in hand perfusion scintigraphic features between primary RP and HAVS. These results suggest that the underlying pathophysiology of the two diseases differs; thus, different criteria should be applied for their evaluation.

\section{CLINICAL FEATURES, OUTCOMES AND RISK FACTORS FOR THE DEVELOPMENT OF POSTERIOR REVERSIBLE ENCEPHALOPATHY SYNDROME (PRES) IN THAI PATIENTS WITH SYSTEMIC LUPUS ERYTHEMATOSUS} (SLE)

${ }^{1} \mathrm{U}$ Damrongpipatkul, ${ }^{1} \mathrm{~N}$ Kasitanon, ${ }^{2} \mathrm{~K}$ Oranratanachai, ${ }^{1} \mathrm{~W}$ Louthrenoo*. ${ }^{1}$ Faculty of Medicine Chiang Mai University, Division of Rheumatology Department of Internal Medicine, Chiang Mai, Thailand; ${ }^{2}$ Faculty of Medicine Chiang Mai University, Division of Neuroimaging and Interventional Radiology Department of Radiology, Chiang Mai, Thailand

\subsection{6/lupus-2017-000215.372}

Background and aims PRES in SLE is increasingly recognised. This study aimed at determining the prevalence, clinical features, brain imaging findings, risk factors, and outcomes of PRES in Thai SLE patients.

Methods SLE patients with PRES seen between 1 January 1986 and 31 August 2013 were identified. Controls were matched with hospital number and disease duration to cases (ratio, 1:4). Clinical features, brain imaging patterns, risk factors, treatment and outcome of PRES were determined.

Results Of 1141 SLE patients, 26 PRES episodes occurred 20 in females (prevalence 1.8\%). Mean $\pm \mathrm{SD}$ age at diagnosis and disease duration was $29.3 \pm 13.1$ and $2.8 \pm 3.4$ years, respectively. Among the 26 episodes, 24 (92.3\%) had seizure, 14 $(53.8 \%)$ headache, $9(34.6 \%)$ fever and vomiting and 8 (30.8\%) visual disturbance. All of them had acutely elevated blood pressure. 20 and 23 patients had active lupus nephritis (LN) within 3 months prior to and at PRES onset, respectively. Dominant parietal-occipital pattern was the most common brain imaging abnormality. 22 episodes improved with blood pressure control. Immunosuppressive therapy was given for active disease in 8 episodes. Anti-convulsive therapy could be discontinued in 21 of 22 episodes (median duration 3 months). Auto-immune hemolytic anaemia (AIHA) and LN were PRES risk factors (OR 6.55, 95\% CI 1.09-39.39, $\mathrm{p}=0.04$ and $\mathrm{OR} 3.06,95 \%$ CI 1.12-8.39, $\mathrm{p}=0.03$, respectively). 6 patients $(23.1 \%)$ died during PRES episodes. The mortality rate in SLE patients with PRES was significantly higher than those without $(30 \%$ vs. $10 \%, \mathrm{p}<0.001)$.

Conclusions The mortality rate was high in Thai SLE with PRES. AIHA and LN were risk factors for PRES.

\section{ASSOCIATION BETWEEN DISEASE ACTIVITY, QUALITY OF LIFE AND QUALITY OF SLEEP IN PATIENTS WITH SYSTEMIC LUPUS ERYTHEMATOSUS (SLE)}

'D Monova, ${ }^{2} S$ Monov*. 'Medical University - Sofia- Medical Institute, Department of Internal Diseases, Sofia, Bulgaria; ${ }^{2}$ Medical University - Sofia, Department of Internal Diseases- Clinic of Rheumatology, Sofia, Bulgaria

10.1136/lupus-2017-000215.373
Background and aims The aim of this study was to examine the association of SLE disease activity, quality of life and quality of sleep in patients diagnosed with SLE.

Methods 132 SLE patients with a confirmed diagnosis of SLE, according ACR classification criteria, were enrolled in this study. The patients completed the following questionnaires: the Pittsburg Sleep Quality Index (PSQI), the 12 item Short Form Health survey (SF-12), the Lupus Patient-Reported Outcome tool (PupusPRO), SLE Quality of Life Questionnaire (LQoL). Clinical information, including the SLE Disease Activity Index (SLEDAI), was obtained from medical records. Student's t-test, ANOVA, Pearson correlation measured were used in statistical analysis.

Results The majority of the participants $(84,4 \%)$ had sleep disturbances (PSQI >5). Total PSQI score was weakly associated with all of the SF-12 subcategories and showed weak to moderate associations with LupusPRO subcategories $(\mathrm{r}<0,05)$, except for medication $(r<0,2)$. "Sleep efficiency" was weakly associated with "physical health", "physical function", and "paim" in the SF-12 and LupusPRO. "Sleep quality" and "sleep disturbances" were weakly associated with "pain" and the "emotional" and "mental" subcategories in the SF-12 and LupusPRO. SLE QoL was significantly higher in patients with good sleep.

Conclusions We found that quality of sleep, especially "sleep efficiency", was poor for the majority of patients with SLE. Quality of sleep was associated with various aspects of quality of life, especially pain, vitality, and emotional health. Management of pain and emotional health may be important for improving quality of sleep in SLE patients.

\section{ANA NEGATIVE RENAL LIMITED LUPUS NEPHRITIS -A RARE ENTITY}

${ }^{1} \mathrm{SP}$ Nagaraju*, ${ }^{1} \mathrm{RP}$ Attur, ${ }^{1} \mathrm{D}$ Rangaswamy, ${ }^{2} \mathrm{SL}$ Koulmane Laxminarayana, ${ }^{1} \mathrm{SP}$ Rao, ${ }^{1} \mathrm{~S}$ Kaza, ${ }^{1} \mathrm{~K}$ Saraf, 'S Shenoy, 'M Bhojaraja, ${ }^{1} \mathrm{~A}$ Rangaswamy, ${ }^{3} \mathrm{~V}$ Mahesha. ${ }^{1}$ Kasturba Medical college- Manipal University, Nephrology, Manipal, India; ${ }^{2}$ Kasturba Medical college- Manipal University, pathology, Manipal, India; ${ }^{3}$ Manipal HospitalBangalore, Pathology, Bangalore, India

\subsection{6/lupus-2017-000215.374}

Background and aims Antinuclear antibodies (ANA) in serum is considered a decisive diagnostic test for SLE. ANA negative SLE is a subgroup of SLE that is infrequently recognised. We report an unusual case of seronegative SLE which presented

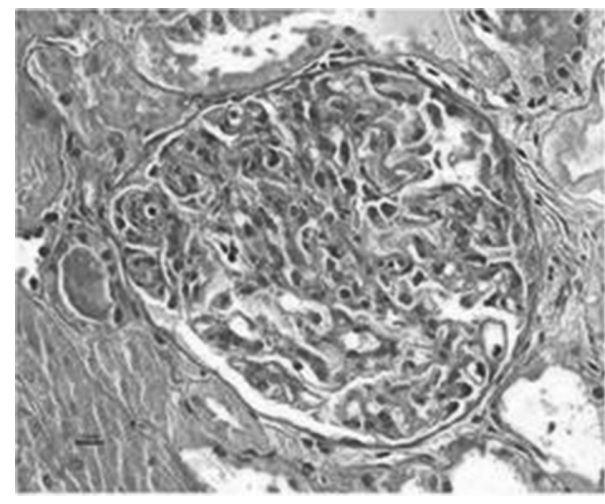

Abstract 374 Figure 1 